\title{
ON THE SEMILATTICE OF IDEMPOTENTS OF A FREE INVERSE MONOID
}

\author{
by PEDRO V. SILVA
}

(Received 12th November 1991)

\begin{abstract}
Some new concepts are introduced, in particular that of a unique factorization semilattice. Necessary and sufficient conditions are given for two principal ideals of the semilattice of idempotents of a free inverse monoid $F I M(X)$ to be isomorphic and some properties of the Munn semigroup of $E[F I M(X)]$ are obtained. Some results on the embedding of semilattices in $E[F I M(X)]$ are also obtained.
\end{abstract}

1991 Mathematics subject classification: 20M05

\section{Preliminaries}

The general terminology and notation are those of Petrich [6].

Let $S$ be a semigroup. We say that $S$ is a semilattice if the following conditions hold:

$$
\begin{gathered}
\forall e \in S, e^{2}=e ; \\
\forall e, f \in S, e f=f e .
\end{gathered}
$$

Let $E$ be a semilattice. The natural partial order on $E$ is defined by

$$
e \leqq f \Leftrightarrow e=e f \text {. }
$$

Suppose that $e, f \in E$ are such that $e<f$ and the condition

$$
e \leqq g<f \Rightarrow e=g
$$

holds for every $g \in E$. Then we say that $f$ covers $e$ and we denote this fact by $e<f$. For every $f \in E$, we define $\operatorname{Cov}(f)=\{e \in E: e<f\}$.

For every $e \in E$, we say that $E e=\{f e: f \in E\}$ is the principal ideal of $E$ generated by $e$.

Now let $S$ be an inverse semigroup. The subset of all idempotents of $S$ is a semilattice, usually denoted by $E(S)$, and so $E(S)$ is said to be the semilattice of idempotents of $S$.

Let $X$ be a nonempty set. We define $X^{-1}=\left\{x^{-1}: x \in X\right\}$ to be a set such that

$$
\begin{gathered}
X \cap X^{-1}=\varnothing ; \\
\forall x_{1}, x_{2} \in X, x_{1}^{-1}=x_{2}^{-1} \Rightarrow x_{1}=x_{2} .
\end{gathered}
$$


Moreover, we define $\left(x^{-1}\right)^{-1}=x$ for every $x \in X$. Now let $\left(X \cup X^{-1}\right)^{*}$ denote the free monoid on $X \cup X^{-1}[2, \S 9.1]$, and let

$$
R_{X}=\left(X \cup X^{-1}\right)^{*}\left[\bigcup_{x \in X \cup X^{-1}}\left(X \cup X^{-1}\right)^{*} x x^{-1}\left(X \cup X^{-1}\right)^{*}\right]
$$

We define a partial order $\leqq_{l}$ on $R_{X}$ by

$$
u \leqq l v \Leftrightarrow v \in u R_{X}
$$

A subset $A$ of $R_{X}$ is said to be left closed if

$$
\forall v \in A \forall u \in R_{X}, u \leqq \imath v u \in A \text {. }
$$

Finally, let $E_{X}$ denote the set of all finite nonempty left closed si'bsets of $R_{X}$, with the operation described by

$$
A B=A \cup B
$$

and let $F I M(X)$ denote the free inverse monoid on $X[6, \S$ VIII.1.].

Lemma $1.1[5 ; 8]$. Let $X$ be a nonempty set. Then

$$
E_{X} \cong E[F I M(X)]
$$

For the remainder of this paper, we assume that $E_{X}=E[F I M(X)]$. Let $v \in R_{X}$. We define

$$
\bar{v}=\left\{u \in R_{X}: u \leqq{ }_{l} v\right\}
$$

It is immediate that $\bar{v} \in E_{X}$ for every $v \in R_{X}$. It follows easily that, for every $A, B \in E_{X}$,

$$
A \geqq B \Leftrightarrow A \subseteq B,
$$

and for every $A \in E_{X}$,

$$
A=\prod_{u \in A} \bar{u}
$$

\section{Unique factorization semilattices}

In this section we introduce some concepts in semilattice theory and we relate them to $E_{X}$. 
Let $E$ be a semilattice and let $e \in E$. We say that $e$ is irreducible if, for every $f, g \in E$,

$$
e=f g \Rightarrow e=f \text { or } e=g \text {. }
$$

The set of all irreducibles of $E$ is denoted by $\operatorname{Irr}(E)$.

We say that $e$ is prime if, for every $f, g \in E$,

$$
e \geqq f g \Rightarrow e \geqq f \text { or } e \geqq g
$$

Lemma 2.1. Let $E$ be a semilattice and let $e \in E$. Then

$$
\text { e prime } \Rightarrow \text { e irreducible. }
$$

Proof. Suppose that $e$ is prime and suppose that $e=f g$ for some $f, g \in E$. Then $e \leqq f$ and $e \leqq g$. Further, $e \geqq f g$ and so, since $e$ is prime, we have $e \geqq f$ or $e \geqq g$. Hence $e=f$ or $e=g$. Thus $e$ is irreducible.

The semilattice $E$ is said to be a unique factorization semilattice (UFS) if

(i) $E$ is generated by $\operatorname{Irr}(E)$;

(ii) every irreducible is prime.

All these concepts are inspired by well-known concepts for integral domains $[1, \S 5.3]$.

We need some results on UFSs.

Lemma 2.2. Let $E$ denote a UFS. Let $e_{1}, \ldots, e_{n}, f_{1}, \ldots, f_{m} \in \operatorname{Irr}(E)$ be such that $e_{1} \ldots e_{n}=f_{1} \ldots f_{m}$. Then, for every $i \in\{1, \ldots, n\}$, there exists $j \in\{1, \ldots, m\}$ such that $e_{i} \geqq f_{j}$.

Proof. Let $i \in\{1, \ldots, n\}$. Clearly, $e_{i} \geqq f_{1} \ldots f_{m}$. Since $E$ is a UFS, $e_{i}$ is prime and an elementary induction yields $e_{i} \geqq f_{j}$ for some $j \in\{1, \ldots, m\}$.

Lemma 2.3. Let $E$ denote $a U F S$ and let $e \in E$. Then

(i) $\operatorname{Irr}(E e)=e \cdot \operatorname{Irr}(E)$;

(ii) $E e$ is a UFS.

Proof. (i) Let $f \in \operatorname{Irr}(E e)$. Since $E$ is a UFS, there exist $g_{1}, \ldots, g_{n} \in \operatorname{Irr}(E)$ such that $f=g_{1} \ldots g_{n}$. Let $l$ be minimal among the nonempty subsets of $\{1, \ldots, n\}$ with respect to $f=e \prod_{i \in l} g_{i}$. Suppose that $|l|>1$. Since $e g_{i}>f$ for every $i \in l$ and $f=\prod_{i \in l} e g_{i}$, we obtain $f \notin \operatorname{Irr}(E e)$, a contradiction. Hence $|l|=1$ and so $f \in e . \operatorname{Irr}(E)$.

Conversely, let $g \in \operatorname{Irr}(E)$ and suppose that $e g=f f^{\prime}$ for some $f, f^{\prime} \in E e$. We have $e \geqq f \geqq e g$ and $e \geqq f^{\prime} \geqq e g$. But $g \geqq f f^{\prime}$ and since $E$ is a UFS, $g$ is prime, so $g \geqq f$ or $g \geqq f^{\prime}$. Without loss of generality, we can assume that $g \geqq f$. Hence $e g \geqq f$ and so $e g=f$. Thus $e g \in \operatorname{Irr}(E e)$.

(ii) Let $f \in E e$. Since $E$ is a UFS, there exist $g_{1}, \ldots, g_{n} \in \operatorname{Irr}(E)$ such that $f=g_{1} \ldots g_{n}$. 
Therefore $f=e f=e g_{1} \ldots g_{n}=\left(e g_{1}\right) \ldots\left(e g_{n}\right)$. By (i), $e g_{i} \in \operatorname{Irr}(E e)$ for every $i \in\{1, \ldots, n\}$. Thus $E e$ is generated by $\operatorname{Irr}(E e)$.

Now let $h \in \operatorname{Irr}(E e)$ and let $a, b \in E e$. Suppose that $h \geqq a b$. By (i), we have $h=e g$ for some $g \in \operatorname{Irr}(E)$. Hence $g \geqq a b$ and so, since $g$ is prime, $g \geqq a$ or $g \geqq b$. We can assume that $g \geqq a$. Since $e \geqq a$, we have $h=e g \geqq a$. Thus $h$ is prime and the lemma is proved.

Lemma 2.4. Let $E$ be a UFS. Let $e, f, g \in \operatorname{Irr}(E)$ be such that $f \succ e$ and $g \succ e$. Then $f=g$.

Proof. Since $f \geqq e$ and $g \geqq e$, we have $f g \geqq e$. Suppose that $f g=e$. Since $e \in \operatorname{Irr}(E)$, we must have $e=f$ or $e=g$, a contradiction. Hence $f g>e$. Now $f \geqq f g>e$ and so, since $f>e$, we have $f=f g$. Therefore $f \leqq g$ and so $f=g$.

We say that a semilattice $E$ is upper finite if the sets $\{f \in E: f \geqq e\}$ are finite for all $e \in E$.

The next lemmas state some properties of $E_{X}$.

Lemma 2.5. Let $X$ be a nonempty set. Then

(i) $\operatorname{Irr}\left(E_{X}\right)=\left\{\bar{w}: w \in R_{X}\right\}$;

(ii) $E_{X}$ is a UFS;

(iii) $E_{X}$ is upper finite.

Proof. Let $A \in \operatorname{Irr}\left(E_{X}\right)$. By (1.2), we have $A=\prod_{u \in A} \bar{u}$. Since $A \in \operatorname{Irr}\left(E_{X}\right)$, we have $A=\bar{u}$ for some $u \in A$. Therefore $\operatorname{Irr}\left(E_{X}\right) \subseteq\left\{\bar{w}: w \in R_{X}\right\}$.

Now suppose that $w \in R_{X}$. We prove that $\bar{w}$ is prime. Suppose that $\bar{w} \geqq A B$ for some $A, B \in E_{X}$. By (1.1), we have $w \in \bar{w} \subseteq A B=A \cup B$. We can assume that $w \in A$. But $A$ is left closed and so $\bar{w} \subseteq A$. Thus $\bar{w} \geqq A$, by (1.1), and so $\bar{w}$ is prime.

By Lemma 2.1, this implies $\bar{w}$ irreducible and so (i) is proved. Moreover, it follows that every irreducible of $E_{X}$ is prime. By (i) and (1.2), $\operatorname{Irr}\left(E_{X}\right)$ generates $E_{X}$ and so $E_{X}$ is a UFS.

It follows easily from (1.1) that $E_{X}$ is upper finite.

Lemma 2.6. Let $X$ be a nonempty set. Let $A \in E_{X}$ and let $B \in\left[\operatorname{Irr}\left(E_{X} A\right)\right] \backslash\{A\}$. Then there exists a unique $C \in \operatorname{Irr}\left(E_{X} A\right)$ such that $B \prec C$.

Proof. By Lemma 2.5(ii), $E_{X}$ is a UFS and so, by Lemmas 2.3 and 2.5(i), we have $B=A \bar{u}$ for some $u \in R_{X}$, Since $B \neq A$, we have $u \notin A$. In particular, $u \neq 1$ and so we can define $v \in R_{X}$ to be the maximal proper prefix of $u$. Let $C=A \bar{v}$. By Lemmas 2.3 and 2.5, $C \in \operatorname{Irr}\left(E_{X} A\right)$. Since $|C|=|B|-1$, we have $B \prec C$. The uniqueness of $C$ follows from Lemma 2.4, replacing $E$ by $E_{X} A$ and $e$ by $B$. 


\section{Principal ideals}

In this section we shall obtain necessary and sufficient conditions for two principal ideals of $E_{X}$ to be isomorphic.

Lemma 3.1. Let $X$ be a nonempty set and let $A \in E_{X}$. Then

$$
|\operatorname{Cov}(A)|= \begin{cases}2|A|(|X|-1)+2 & \text { if } X \text { is finite } \\ |X| & \text { if } X \text { is infinite }\end{cases}
$$

Proof. We assume that $X$ is finite, the other case being obvious. We use induction on $|A|$.

Suppose that $|A|=1$. Then $A=\{1\}$ and so $\operatorname{Cov}(A)=\left\{\{1, x\}: x \in X \cup X^{-1}\right\}$. Hence $|\operatorname{Cov}(A)|=2|X|$ and the lemma holds.

Now suppose that the lemma holds for every $B \in E_{X}$ such that $|B| \leqq n$, with $n \in \mathbb{N}$. Let $A \in E_{X}$ be such that $|A|=n+1$. Let $v \in A$ have maximal length. Since $|A|>1$, we have $|v|>1$. Let $y \in X \cup X^{-1}$ denote the last letter of $v$. Let $A^{\prime} \in E_{X}$ and suppose that $A^{\prime} \in \operatorname{Cov}(A)$. Since $\left|A^{\prime} \backslash A\right|=1$, we can define $u\left(A^{\prime}\right)$ to be the single element of $A^{\prime} \backslash A$. We define $\Gamma=\left\{A^{\prime} \in \operatorname{Cov}(A): v \leqq{ }_{l} u\left(A^{\prime}\right)\right\}$ and $\Lambda=[\operatorname{Cov}(A)] \backslash \Gamma$.

Let $A^{\prime} \in \Gamma$. Since $|v|$ is maximal in $A$ and $\left|A^{\prime} \backslash A\right|=1$, it follows that $u\left(A^{\prime}\right)=v x$ for some $x \in X \cup X^{-1}$. Since $y$ is the last letter of $v$ and $u\left(A^{\prime}\right) \in R_{X}$, we have $x \neq y^{-1}$ and so $\Gamma=\left\{A \cup\{v x\}: x \in\left(X \cup X^{-1}\right) \backslash\left\{y^{-1}\right\}\right\}$. Hence $|\Gamma|=2|X|-1$.

Let $A_{0}=A \backslash\{v\}$. Since $|v|$ is maximal in $A$ and $|v|>1$, we have $A_{0} \in E_{X}$. We define a map $\varphi: \Lambda \rightarrow \operatorname{Cov}\left(A_{0}\right)$ as follows. Suppose that $A^{\prime} \in \Lambda$. Let $A_{0}^{\prime}=A^{\prime} \backslash\{v\}$. Since $v \$ \imath\left(A^{\prime}\right)$, it follows that $v$ must be maximal in $A^{\prime}$ for $\leqq_{l}$. Hence $A_{0}^{\prime} \in E_{X}$. It is clear that $A_{0}^{\prime} \in \operatorname{Cov}\left(A_{0}\right)$ and so we can define $A^{\prime} \varphi=A_{0}^{\prime}$. Moreover, $\varphi$ is injective and $\left[\operatorname{Cov}\left(A_{0}\right)\right] \backslash$ $(\Lambda \varphi)=\{A\}$. Hence $|\Lambda|=\left|\operatorname{Cov}\left(A_{0}\right)\right|-1$. Using the induction hypothesis, we obtain $|\Lambda|=2\left|A_{0}\right|(|X|-1)+2-1=2 n(|X|-1)+1$. Thus $|A|=|\Gamma|+|\Lambda|=2|X|-1+2 n(|X|-1)+$ $1=2(n+1)(|X|-1)+2=2|A|(|X|-1)+2$ and the result follows by induction.

We must introduce some new concepts and notation.

Let $A \in E_{X}$ and let $m=|A|$. For all $k \in \mathbb{N}^{0}$, we define $\operatorname{Irr}_{m+k}\left(E_{X} A\right)=\left\{B \in \operatorname{Irr}\left(E_{X} A\right):|B|=\right.$ $m+k\}$. Surely, $\operatorname{Irr}\left(E_{X} A\right)=\bigcup_{k \geqq 0} \operatorname{Irr}_{m+k}\left(E_{X} A\right)$. Moreover, $\operatorname{Irr}_{m}\left(E_{X} A\right)=\{A\}$ and $\operatorname{Irr}_{m+1}\left(E_{X} A\right)=\operatorname{Cov}(A)$. For every $B \in \operatorname{Irr}\left(E_{X} A\right)$, we define $[B]_{A}=\left\{C \in \operatorname{Irr}\left(E_{X} A\right): C \prec B\right\}$. Suppose that $B \neq A$. By Lemmas 2.3 and 2.5 , we have $B=A \bar{u}$ for some $u \in R_{X} \backslash A$. It follows easily that $[B]_{A}=\left\{A \bar{v}: v \in R_{X}\right.$ and $v=u x$ for some $\left.x \in X \cup X^{-1}\right\}$. Thus

$$
\left|[B]_{A}\right|= \begin{cases}2|X|-1 & \text { if } X \text { is finite } \\ |X| & \text { if } X \text { is infinite. }\end{cases}
$$

Now we obtain a criterion for isomorphism.

Lemma 3.2. Let $X$ be a nonempty set and let $A, B \in E_{X}$. Then 


$$
E_{X} A \cong E_{X} B \Leftrightarrow|\operatorname{Cov}(A)|=|\operatorname{Cov}(B)|
$$

Proof. Suppose that $\Phi: E_{X} A \rightarrow E_{X} B$ is an isomorphism. We certainly have $A \Phi=B$. Let $A^{\prime} \in \operatorname{Cov}(A)$. Since $\Phi$ is injective, we have $A^{\prime} \Phi<B$. Suppose that $A^{\prime} \Phi<B^{\prime}<B$ for some $B^{\prime} \in E_{X} B$. Let $A^{\prime \prime}=B^{\prime} \Phi^{-1}$. It follows easily that $A^{\prime}<A^{\prime \prime}<A$, in contradiction with $A^{\prime} \in \operatorname{Cov}(A)$. Hence no such $B^{\prime}$ exists and so $A^{\prime} \Phi \in \operatorname{Cov}(B)$. Thus $[\operatorname{Cov}(A)] \Phi \subseteq \operatorname{Cov}(B)$. Similarly, we obtain $[\operatorname{Cov}(B)] \Phi^{-1} \subseteq \operatorname{Cov}(A)$. Hence $[\operatorname{Cov}(A)] \Phi=\operatorname{Cov}(B)$ and so $|\operatorname{Cov}(A)|$ $=|\operatorname{Cov}(B)|$.

Conversely, suppose that $|\operatorname{Cov}(A)|=|\operatorname{Cov}(B)|$. Suppose that $m=|A|$ and $n=|B|$. For every $k \in \mathbb{N}^{0}$, we define a bijection $\varphi_{k}: \operatorname{Irr}_{m+k}\left(E_{X} A\right) \rightarrow \operatorname{Irr}_{n+k}\left(E_{X} B\right)$ as follows.

Consider $k=0$. Since $\operatorname{Irr}_{m}\left(E_{X} A\right)=\{A\}$ and $\operatorname{Irr}_{n}\left(E_{X} B\right)=\{B\}$, we define $A \varphi_{0}=B$.

Now suppose that $\varphi_{k}$ is defined for some $k \in \mathbb{N}^{0}$. Let $C \in \operatorname{Irr}_{m+k}\left(E_{X} A\right)$. Suppose first that $k=0$. Then $C=A$ and $C \varphi_{k}=B$ and so $\left|[C]_{A}\right|=|\operatorname{Cov}(A)|=|\operatorname{Cov}(B)|=\left|\left[C \varphi_{k}\right]_{B}\right|$. Suppose now that $k>0$. By (3.1), we obtain $\left|[C]_{A}\right|=\left|\left[C \varphi_{k}\right]_{B}\right|$ as well. Whatever the case, we can define a bijection $\psi_{C}:[C]_{A} \rightarrow\left[C \varphi_{k}\right]_{B}$ for every $C \in \operatorname{Irr}_{m+k}\left(E_{X} A\right)$. Since $\operatorname{Irr}_{m+k+1}\left(E_{X} A\right)=\bigcup_{C_{\in \operatorname{Irr} m+k}\left(E_{X} A\right)}[C]_{A}$ and $\operatorname{Irr}_{n+k+1}\left(E_{X} B\right)=\bigcup_{D \in \operatorname{Irr}_{n+k}\left(E_{X} B\right)}[D]_{B}$, there is a unique map $\varphi_{k+1}: \operatorname{Irr}_{m+k+1}\left(E_{X} A\right) \rightarrow \operatorname{Irr}_{n+k+1}\left(E_{X} B\right)$ such that, for every $C \in \operatorname{Irr}_{m+k}\left(E_{X} A\right),\left.\varphi_{k+1}\right|_{[C]_{A}}=\psi_{C}$. Since $\varphi_{k}$ is bijective and every $\psi_{C}$ is bijective, it follows that $\varphi_{k+1}$ is bijective as well. Next, we define $\varphi: \operatorname{Irr}\left(E_{X} A\right) \rightarrow \operatorname{Irr}\left(E_{X} B\right)$ to be the unique bijection such that $\left.\varphi\right|_{\operatorname{Irr}_{m+k}\left(E_{X} A\right)}=\varphi_{k}$ for every $k \in \mathbb{N}^{0}$.

We prove that, for every $C, D \in \operatorname{Irr}\left(E_{X} A\right)$,

$$
C \prec D \Leftrightarrow C \varphi \prec D \varphi
$$

Suppose that $C \prec D$. Then $C \in[D]_{A}$ and so $C \varphi=C \psi_{D} \in[D \varphi]_{B}$. Hence $C \varphi \prec D \varphi$.

Conversely, suppose that $C \varphi \prec D \varphi$. It is immediate that $C \neq A$. By Lemma 2.6, there exists a unique $C^{\prime} \in \operatorname{Irr}\left(E_{X} A\right)$ such that $C \prec C^{\prime}$. It follows from above that $C \varphi \prec C^{\prime} \varphi$. By Lemma 2.4, with $E$ replaced by $E_{X} B$ and $e$ replaced by $C \varphi$, we obtain $D \varphi=C^{\prime} \varphi$. Since $\varphi$ is bijective, it follows that $D=C^{\prime}$ and so $C \prec D$. Thus (3.2) holds.

Since $E_{X}$ is upper finite, it follows easily from (3.2) that, for every $C, D \in \operatorname{Irr}\left(E_{X} A\right)$,

$$
C \leqq D \Leftrightarrow C \varphi \leqq D \varphi
$$

Suppose now that $C_{1} \ldots C_{r}=D_{1} \ldots D_{s}$, with $C_{1}, \ldots, C_{r}, D_{1}, \ldots, D_{s} \in \operatorname{Irr}\left(E_{X} A\right)$. Let $i \in\{1, \ldots, r\}$. By Lemmas 2.2 and $2.3\left(\right.$ ii), there exists $j \in\{1, \ldots, s\}$ such that $C_{i} \geqq D_{j}$. By (3.3), we have $C_{i} \varphi \geqq D_{j} \varphi$ and so $C_{1} \varphi \ldots C_{r} \varphi \geqq D_{1} \varphi \ldots D_{s} \varphi$. Similarly, we obtain $D_{1} \varphi \ldots D_{s} \varphi \geqq C_{1} \varphi \ldots C_{r} \varphi$ and so $C_{1} \varphi \ldots C_{r} \varphi=D_{1} \varphi \ldots D_{s} \varphi$. A similar argument shows that $C_{1} \varphi \ldots C_{r} \varphi=D_{1} \varphi \ldots D_{s} \varphi$ implies $C_{1} \ldots C_{r}=D_{1} \ldots D_{s}$ and so we can define an injecive map $\Phi: E_{X} A \rightarrow E_{X} B$ as follows. Let $C \in E_{X} A$. By Lemma 2.3 , we can write $C=C_{1} \ldots C_{r}$ for some $C_{1}, \ldots, C_{r} \in \operatorname{Irr}\left(E_{X} A\right)$. Then we define $C \Phi=C_{1} \varphi \ldots C_{r} \varphi$.

We show that $\Phi$ is an isomorphism.

Let $C \in E_{X} B$. By Lemma 2.3(ii), there exist $C_{1}, \ldots, C_{r} \in \operatorname{Irr}\left(E_{X} B\right)$ such that $C=C_{1} \ldots C_{r}$. Since $\varphi$ is bijective, there exist $D_{1}, \ldots, D_{r} \in \operatorname{Irr}\left(E_{X} A\right)$ such that $C_{i}=D_{i} \varphi$ for every $i \in\{1, \ldots, r\}$. Thus $C=C_{1} \ldots C_{r}=D_{1} \varphi \ldots D_{r} \varphi=\left(D_{1} \ldots D_{r}\right) \Phi$ and so $\Phi$ is surjective. 
Let $C, D \in E_{X} A$. Suppose that $C=C_{1} \ldots C_{r}$ and $D=D_{1} \ldots D_{s}$ for some $C_{1}, \ldots, C_{r}, D_{1}, \ldots, D_{s} \in \operatorname{Irr}\left(E_{X} A\right)$. Then $C \Phi . D \Phi=\left(C_{1} \ldots C_{r}\right) \Phi .\left(D_{1} \ldots D_{s}\right) \Phi=C_{1} \varphi \ldots$ $C_{r} \varphi D_{1} \varphi \ldots D_{s} \varphi=\left(C_{1} \ldots C_{r} D_{1} \ldots D_{s}\right) \Phi=(C D) \Phi$. Thus $\Phi$ is a homomorphism and the lemma is proved.

We note that every isomorphism $\Phi: E_{X} A \rightarrow E_{X} B$ must induce bijections between $\operatorname{Irr}_{m+k}\left(E_{X} A\right)$ and $\operatorname{Irr}_{n+k}\left(E_{X} B\right)$ and satisfy (3.2).

Now Lemmas 3.1 and 3.2 yield:

Theorem 3.3. Let $X$ be a nonempty set and let $A, B \in E_{X}$.

(i) If $X$ is infinite or $|X|=1$, then $E_{X} A \cong E_{X} B$.

(ii) If $X$ is finite and $|X|>1$, then

$$
E_{X} A \cong E_{X} B \Leftrightarrow|A|=|B|
$$

A semilattice in which all the principal ideals are isomorphic is said to be uniform. It follows from Theorem 3.3 that, if $X$ is infinite or $|X|=1$, then $E_{X}$ is uniform.

\section{The Munn semigroup}

We can use the results obtained in Section 3 to get information about the Munn semigroup [4] of the semillatice $E_{X}$.

Let $E$ be a semilattice and let $U=\{(e, f) \in E \times E: E e \cong E f\}$. For every $(e, f) \in U$, let $T_{e, f}$ denote the set of all isomorphisms from $E e$ onto $E f$. The Munn semigroup of $E$ is defined to be $T_{E}=\bigcup_{(e, f) \in U} T_{e, S}$, with the usual composition of relations [3, §V.4]. This is an inverse semigroup and $E\left(T_{E}\right)=\left\{1_{E e}: e \in E\right\}$ is isomorphic to $E$. It follows easily from the definition that, for every $e, f \in E, 1_{E e} \mathscr{D}=1_{E f} \mathscr{D}$ if and only if $(e, f) \in U$.

Theorem 4.1. Let $X$ be a nonempty set. Then $T_{E_{X}}$ is E-unitary.

Proof. Let $A, B, C \in E_{X}$ and let $\Phi: E_{X} A \rightarrow E_{X} B$ be an isomorphism. Suppose that $1_{E_{X} c} . \Phi \in E\left(T_{E_{X}}\right)$. We want to prove that $\Phi \in E\left(T_{E_{X}}\right)$. We have that $1_{E_{X} c} . \Phi$ is the restriction of $\Phi$ to the semilattice $\left(E_{X} C\right) \cap\left(E_{X} A\right)$, that is, $E_{X} C A$. Therefore we have $\left.\Phi\right|_{E_{X} C_{A}}=1_{E_{X} C_{A}}$ and we must show that $\Phi=1_{E_{X} A}$.

Suppose that $\Phi \neq 1_{E_{X} A}$. We show that

$$
\exists D \in \operatorname{Irr}\left(E_{X}\right) \text { such that } D \neq A \text { and }(A D) \Phi \neq B D .
$$

Assume first that $A=B$. Since $\Phi \neq 1_{E_{X} A}$, there exists $U \in E_{X} A$ such that $U \Phi \neq U$. Since $A \Phi=B=A$, we have $U \neq A$ and so we can write $U=A D_{1} \ldots D_{n}$ for some $D_{i} \in \operatorname{Irr}\left(E_{X}\right)$, with $D_{i}$ 聿 $A, i \in\{1, \ldots, n\}$. It follows that $D_{i} \Phi \neq D_{i}$ for some $i$ and so (4.1) holds.

Now assume that $A \neq B$. Since $\operatorname{Cov}(A) \subseteq \operatorname{Irr}\left(E_{X} A\right)$, and by Lemma 2.3(i), there exist 
$\left\{D_{i}: i \in l\right\} \subseteq \operatorname{Irr}\left(E_{X}\right)$ such that $\operatorname{Cov}(A)=\left\{A D_{i}: i \in l\right\}$. Suppose that $\left(A D_{i}\right) \Phi=B D_{i}$ for every $i \in l$. Since $[\operatorname{Cov}(A)] \Phi=\operatorname{Cov}(B)$, we have $\operatorname{Cov}(B)=\left\{B D_{i}: i \in l\right\}$.

Suppose that $A \notin B$. Let $u \in A \backslash B$. Let $u^{\prime}$ denote the maximum prefix of $u$ contained in $B$ and suppose that $u=u^{\prime} x u^{\prime \prime}$, with $x \in X \cup X^{-1}$ and $u^{\prime \prime} \in R_{X}$. Then $\overline{B u^{\prime} x} \in \operatorname{Cov}(B)$ and so $\overline{B u^{\prime} x}=B D_{i}$ for some $i \in l$. Since $\overline{u^{\prime} x}, D_{i} \in \operatorname{Irr}\left(E_{X}\right)$, we show easily that $\overline{u^{\prime} x}=D_{i}$. In fact, $D_{i} \geqq B \overline{u^{\prime} x}$ and $D_{i} \geq B$ together imply $D_{i} \geqq \overline{u^{\prime} x}$. Similarly, $\overline{u^{\prime} x} \geqq D_{i}$ and so $\overline{u^{\prime} x}=D_{i}$. However, $\overline{u^{\prime} x} \geqq A$, a contradiction. Thus $A \subseteq B$. Similarly, we obtain $B \subseteq A$ and so $A=B$, a contradiction. Therefore $\left(A D_{i}\right) \Phi \neq B D_{i}$ for some $i \in l$ and so (4.1) holds.

Now suppose that $D \in \operatorname{Irr}\left(E_{X}\right)$ is such that $D \neq A$ and $(A D) \Phi \neq B D$. Let $D^{\prime} \in \operatorname{Irr}\left(E_{X}\right)$ be such that $D^{\prime} \prec D$. By Lemma 2.3(i), $A D \in \operatorname{Irr}\left(E_{X} A\right)$. Hence $(A D) \Phi \in \operatorname{Irr}\left(E_{X} B\right)$ and so, by Lemma 2.3(i), $(A D) \Phi=B U$ for some $U \in \operatorname{Irr}\left(E_{X}\right)$. Since $D \neq A$, we have $U$ $D^{\prime} A$. Hence $A D^{\prime}<A D$ and so $\left(A D^{\prime}\right) \Phi<(A D) \Phi$. Similarly, $\left(A D^{\prime}\right) \Phi=B U^{\prime}$ for some $U^{\prime} \in \operatorname{Irr}\left(E_{X}\right)$. Since $U$ is prime, $B U^{\prime}<B U$ and $U \neq B$, we have $U^{\prime}<U$. If $U^{\prime}<U^{\prime \prime}<U$ for some $U^{\prime \prime} \in E_{X}$, then $U^{\prime \prime} \in \operatorname{Irr}\left(E_{X}\right), U^{\prime \prime} B$ and it follows easily that $B U^{\prime}<B U^{\prime \prime}<B U$, a contradiction. Hence $U^{\prime} \prec U$. Now suppose that $B U^{\prime}=B D^{\prime}$. Since $U^{\prime}, D^{\prime} \in \operatorname{Irr}\left(E_{X}\right)$ and $U^{\prime} B$, it follows easily that $U^{\prime}=D^{\prime}$. But $U^{\prime} \prec U$ and $D^{\prime} \prec D$, so, by Lemma 2.4 , we have $U=D$, a contradiction. Hence $B U^{\prime} \neq B D^{\prime}$, that is, $\left(A D^{\prime}\right) \Phi \neq B D^{\prime}$ and so (4.1) holds for $D \in \operatorname{Irr}\left(E X_{X}\right)$ with arbitrary large cardinal. In particular, we can assume that $|D|>|A B C|$. Suppose that $(A D) \Phi=B U$, with $U \in \operatorname{Irr}\left(E_{x}\right)$. Then $C A D=(C A D) \Phi=(C A) \Phi .(A D) \Phi=$ $C A B U$. Therefore $D \geqq C A B U$. Since $|D|>|C A B|$, we have $D \geq C A B$. Then, since $D$ is prime, we get $D \geqq U$. Hence $|U| \geqq|D|>|A B C| \geqq|C A|$ and so $U \geqq C A$. But $U \geqq C A D$ and so, since $U$ is prime, $U \geqq D$. Therefore $U=D$, a contradiction. Hence $\Phi=1_{E_{X} A}$ and so $T_{E_{X}}$ is E-unitary.

Let $M$ be an inverse monoid. We say that $M$ is bisimple if

$$
\forall e, f \in E(M), e \mathscr{D}=f \mathscr{D} \text {. }
$$

We say that $M$ is completely semisimple if

$$
\forall e, f \in E(M), e \mathscr{D}=f \mathscr{D} \Rightarrow e \nless f .
$$

Theorem 4.2. Let $X$ be a nonempty set. Then

(i) $T_{E_{X}}$ is bisimple if and only if $X$ is infinite or $|X|=1$;

(ii) $T_{E_{X}}$ is completely semisimple if and only if $X$ is finite and $|X|>1$.

Proof. (i) Let $A, B \in A_{X}$. Since $1_{E_{X} A} \mathscr{D}=1_{E_{X} B} \mathscr{D}$ is equivalent to $E_{X} A \cong E_{X} B$, we have that $T_{E_{X}}$ is bisimple if and only if $E_{X}$ is uniform, and Theorem 3.3 yields the result.

(ii) Suppose that $X$ is infinite or $|X|=1$. Let $A, B \in E_{X}$ be such that $A>B$. We have that $1_{E_{X} A} \mathscr{D}=1_{E_{X} B} \mathscr{D}$ and $1_{E_{X} A}>1_{E_{X} B}$, so $T_{E_{X}}$ is not completely semisimple.

Now suppose that $X$ is finite and $|X|>1$. Let $A, B \in E_{X}$ be such that $1_{E_{X} A} \mathscr{D}=1_{E_{X} B} \mathscr{D}$ and $1_{E_{X} A} \leqq 1_{E_{X} B}$. Since $1_{E_{X} A} \mathscr{D}=1_{E_{X} B} \mathscr{D}$, we have $E_{X} A \cong E_{X} B$, and by Theorem 3.3, $|A|=|B|$. Since $1_{E_{X} A} \leqq 1_{E_{X} B}$, we have $A \leqq B$. Clearly, $A \leqq B$ and $|A|=|B|$ together imply $A=B$, so $T_{E_{X}}$ is completely semisimple and the lemma is proved. 


\section{Subsemilattices of $E_{X}$}

The problem of finding necessary and sufficient conditions for a semilattice to be embeddable in $E_{X}$ is still open. In this section, we obtain some results concerning some particular classes of semilattices.

Since the free inverse monoid of countable rank is itself embeddable in any free inverse monoid of rank greater than 1 [7], we will fix $X=\left\{x_{n}: n \in \mathbb{N}\right\}$ throughout this section.

Theorem 5.1. Let $L$ be a finite semilattice. Then $L$ is embeddable in $E_{X}$.

Proof. Let $\varphi: L \rightarrow X$ be an injective map. We define a map $\Phi: L \rightarrow E_{X}$ by $a \Phi=$ $\{1\} \cup\left(L \backslash L^{1} a\right) \varphi$.

We show that $\Phi$ is a homomorphism. Let $a, b \in L$. Since $L^{1} a b=\left(L^{1} a\right) \cap\left(L^{1} b\right)$, we have $(a b) \Phi=\{1\} \cup\left(L \backslash L^{1} a b\right) \varphi=\{1\} \cup\left(L \backslash\left[\left(L^{1} a\right) \cap\left(L^{1} b\right)\right]\right) \varphi=\{1\} \cup\left[\left(L \backslash L^{1} a\right) \cup\left(L \backslash L^{1} b\right)\right] \varphi=$ $\left[\{1\} \cup\left(L \backslash L^{1} a\right) \varphi\right] \cup\left[\{1\} \cup\left(L \backslash L^{1} b\right) \varphi\right]=a \Phi . b \Phi$. Therefore $\Phi$ is a homomorphism.

Now suppose that $a \Phi=b \Phi$. Then $\{1\} \cup\left(L \backslash L^{1} a\right) \varphi=\{1\} \cup\left(L \backslash L^{1} b\right) \varphi$ and so $L^{1} a=L^{1} b$. Hence $a=c b$ for some $c \in L^{1}$, that is, $a \leqq b$. Similarly, $b \leqq a$, hence $a=b$. Thus $\Phi$ is injective and the theorem is proved.

Theorem 5.2. Let $L$ be a countable UFS. Then $L$ is embeddable in $E_{X}$ if and only if $L$ is upper finite.

Proof. Suppose that $L$ is embeddable in $E_{X}$. Clearly, subsemilattices of upper finite semilattices are upper finite. Since $E_{X}$ is upper finite, it follows that $L$ is upper finite.

Conversely, suppose that $L$ is upper finite.

We prove that the elements of $L$ can be written as a sequence $\left(f_{n}: n \in \mathbb{N}\right)$ such that

$$
f_{n} \leqq f_{m} \Rightarrow n \geqq m
$$

Suppose that $L=\left\{e_{n}: n \in \mathbb{N}\right\}$. We define a sequence $\left(A_{n}: n \in \mathbb{N}\right)$ of subsets of $L$ as follows. Assuming that $A_{0}=\varnothing$, we define $A_{n}=\left\{g \in L: g \geqq e_{n}\right\} \backslash\left(A_{0} \cup \ldots \cup A_{n-1}\right)$ for every $n \in \mathbb{N}$. Since $L$ is upper finite, every $A_{n}$ is finite, possibly empty. Moreover, $L=\bigcup_{n \geqq 1} A_{n}$. Now we define the sequence $\left(f_{n}: n \in \mathbb{N}\right)$.

Clearly, $A_{1} \neq \varnothing$. Let $f_{1}$ be maximial in $A_{1}$ for the natural partial order of $L$.

Suppose that $f_{1}, \ldots, f_{k}$ are defined for some $k \in \mathbb{N}$ and suppose that $f_{k} \in A_{n}$. If $A_{n} \backslash\left\{f_{1}, \ldots, f_{k}\right\} \neq \varnothing$, we choose $f_{k+1}$ to be a maximal element of $A_{n} \backslash\left\{f_{1}, \ldots, f_{k}\right\}$. If $A_{n} \backslash\left\{f_{1}, \ldots, f_{k}\right\}=\varnothing$, we choose $f_{k+1}$ to be a maximal element of $A_{n+m}$, where $m=$ $\min \left\{l \in \mathbb{N}: A_{n+1} \neq \varnothing\right\}$. Note that $\left\{l \in \mathbb{N}: A_{n+l} \neq \varnothing\right\}$ is nonempty, since $L$ is countable and $A_{1} \cup \ldots \cup A_{n}$ is finite.

It is immediate that $L=\left\{f_{n}: n \in \mathbb{N}\right\}$ and $\left(f_{n}: n \in \mathbb{N}\right)$ satisfies (5.1).

We define a map $\varphi: L \rightarrow E_{X}$ as follows. Since (5.1) holds, we have $f_{1} \in \operatorname{Irr}(L)$. Let $k \in \mathbb{N}$. The set $B_{k}=\left\{i \in \mathbb{N}: f_{i} \in \operatorname{Irr}(L)\right.$ and $\left.f_{i} \geqq f_{k}\right\}$ is clearly finite. Since $\operatorname{Irr}(L)$ generates $L$, there 
exists some $f_{i} \in \operatorname{Irr}(L)$ such that $f_{i} \geqq f_{k}$ and so $B_{k}$ is nonempty. Since $L$ is a UFS, it is clear that $f_{k}=\prod_{i \in B_{k}} f_{i}$. We define $f_{k} \varphi=\{1\} \cup\left\{x_{i}: i \in B_{k}\right\}$.

We prove that $\varphi$ is a homomorphism. Let $m, n \in \mathbb{N}$ and suppose that $f_{m} f_{n}=f_{k}$. We want to show that $f_{m} \varphi \cdot f_{n} \varphi=f_{k} \varphi$, that is, $B_{m} \cup B_{n}=B_{k}$. Since $f_{k} \leqq f_{m}$ and $f_{k} \leqq f_{n}$, it follows that $B_{m} \cup B_{n} \subseteq B_{k}$. Now suppose that $i \in B_{k}$. Then $f_{i} \in \operatorname{Irr}(L)$ and $f_{i} \geqq f_{k}=f_{m} f_{n}$. Since $L$ is a UFS, $f_{i}$ is prime and so we have $f_{i} \geqq f_{m}$ or $f_{i} \geqq f_{n}$. Hence $i \in B_{m} \cup B_{n}$ and so $B_{k} \subseteq B_{m} \cup B_{n}$. Thus $B_{m} \cup B_{n}=B_{k}$ and $\varphi$ is a homomorphism.

Now suppose that $f_{m} \varphi=f_{n} \varphi$ for some $m, n \in \mathbb{N}$. Then $B_{m}=B_{n}$ and so $f_{m}=\prod_{i \in B_{m}} \cdot f_{i}=$ $\prod_{i \in B_{n}} f_{i}=f_{n}$. Therefore $\varphi$ is injective and the theorem is proved.

We note that these results only yield sufficient conditions for a semilattice to be embeddable in $E_{X}$. We can provide a trivial example of a subsemilattice of $E_{X}$ which is not a UFS. In fact, let $U, V, W, Z \in E_{X}$ be defined by $U=\left\{1, x_{1}, x_{2}\right\}, V=\left\{1, x_{1}, x_{3}\right\}$, $W=\left\{1, x_{2}, x_{3}\right\}$ and $Z=\left\{1, x_{1}, x_{2}, x_{3}\right\}$. Let $N=\{U, V, W, Z\}$. Obviously, $N$ is a subsemilattice of $E_{X}$. However, $N$ is not a UFS, since $U \in \operatorname{Irr}(N), U \geqq V W, U Z V$ and $U$

Theorem 5.3. There exists a countable upper finite semilattice which is not embeddable in $E_{X}$.

Proof. Let $M=\left\{(m, n) \in \mathbb{N}^{0} \times \mathbb{N}^{0}: m \geqq n\right\}$, with multiplication described by

$$
(m, n) \backslash\left(m^{\prime}, n^{\prime}\right)= \begin{cases}\left(m, \min \left\{n, n^{\prime}\right\}\right) & \text { if } m=m^{\prime} \\ \left(\max \left\{m, m^{\prime}\right\}, 0\right) & \text { if } m \neq m^{\prime}\end{cases}
$$

It follows from the definition that the groupoid $M$ is commutative and every element of $M$ is idempotent. We note that $M_{0}=\left\{(m, 0): m \in \mathbb{N}^{0}\right\}$ satisfies $\left(M_{0} M\right) \cup\left(M M_{0}\right) \subseteq M_{0}$. Let $(m, n),\left(m^{\prime}, n^{\prime}\right),\left(m^{\prime \prime}, n^{\prime \prime}\right) \in M$. if $m=m^{\prime}=m^{\prime \prime}$, then $\left[(m, n)\left(m^{\prime}, n^{\prime}\right)\right]\left(m^{\prime \prime}, n^{\prime \prime}\right)=$ $\left(m, \min \left\{n, n^{\prime}, n^{\prime \prime}\right\}\right)=(m, n)\left[\left(m^{\prime}, n^{\prime}\right)\left(m^{\prime \prime}, n^{\prime \prime}\right)\right]$. Otherwise, it follows from the remark on $M_{0}$ that $\left[(m, n)\left(m^{\prime}, n^{\prime}\right)\right]\left(m^{\prime \prime}, n^{\prime \prime}\right)=\left(\max \left\{m, m^{\prime}, m^{\prime \prime}\right\}, 0\right)=(m, n)\left[\left(m^{\prime}, n^{\prime}\right)\left(m^{\prime \prime}, n^{\prime \prime}\right)\right]$. Hence $M$ is associative and so a semilattice.

Let $(m, n),\left(m^{\prime}, n^{\prime}\right) \in M$. It should be clear that $\left(m^{\prime}, n^{\prime}\right) \geqq(m, n)$ implies $m^{\prime} \leqq m$. Since $n^{\prime} \leqq m^{\prime}$, there exist only finitely many $\left(m^{\prime}, n^{\prime}\right) \in M$ such that $\left(m^{\prime}, n^{\prime}\right) \geqq(m, n)$. Hence $M$ is upper finite.

Now suppose that $\varphi: M \rightarrow E_{X}$ is an embedding. Let $k=|(0,0) \varphi|$. Since $(k, k)>$ $(k, k-1)>\ldots>(k, 0)$, we have $(k, k) \varphi>\ldots>(k, 0) \varphi$. Hence $|(k, k) \varphi|<\ldots<|(k, 0) \varphi|$ and so $|(k, 0) \varphi|-|(k, k) \varphi| \geqq k$. Since $|A B| \leqq|A|+|B|-1$ for every $A, B \in E_{x}$, we have $|(k, 0) \varphi|=$ $|(0,0) \varphi .(k, k) \varphi| \leqq|(0,0) \varphi|+|(k, k) \varphi|-1$. Hence $|(0,0) \varphi| \geqq|(k, 0) \varphi|-|(k, k) \varphi|+1 \geqq k+1$, a contradiction. Therefore no such embedding exists.

\section{The Hopf property}

An algebra $A$ is said to be hopfian if the only surjective endomorphisms of $A$ are the automorphisms. 
It is known that $\operatorname{FIM}(X)$ is hopfian if and only if $X$ is finite [5]. However, $E_{X}$ shows different behaviour.

Theorem 6.1. Let $X$ be a nonempty set. Then $E_{X}$ is not hopfian.

Proof. Let $x \in X$ and let

$$
Y=\left\{u \in R_{X}: x^{2} \varliminf_{l} u\right\}
$$

Let $l:\left(X \cup X^{-1}\right)^{*} \rightarrow R_{X}$ denote the map which associates to every $u \in\left(X \cup X^{-1}\right)^{*}$ the corresponding reduced word, obtained by successively deleting all factors of the form $x x^{-1}, x \in X \cup X^{-1}$. Let $A \in E_{X}$. We define $A^{\prime}=(A \backslash Y) \cup\left[x^{-1}(A \cap Y)\right]_{l}$. Obviously, $A^{\prime}$ is finite and nonempty. We show that $A^{\prime}$ is left closed. Let $w \in A^{\prime}$ and let $w^{\prime} \in R_{X}$ with $w^{\prime}<{ }_{l} w$.

Suppose first that $w \in A \backslash Y$. Since $A$ is left closed, we have $w^{\prime} \in A$ and it is clear that $w \notin Y$ implies $w^{\prime} \notin Y$. Hence $w^{\prime} \in A^{\prime}$.

Now suppose that $w \in\left[x^{-1}(A \cap Y)\right] l$. Since $1 \in A \backslash Y$, we can assume that $w^{\prime} \neq 1$. Then there exists some $v \in R_{X}$ such that $x^{2} v \in A$ and $w=x v$. Since $w^{\prime}<_{l} w$ and $w^{\prime} \neq 1$, there exists $v^{\prime} \in R_{X}$ such that $v^{\prime}<_{1} v$ and $w^{\prime}=x v^{\prime}$. Since $A$ is left closed, $x^{2} v^{\prime} \in A$. Hence $w^{\prime}=x v^{\prime}=\left[x^{-1}\left(x^{2} v^{\prime}\right)\right] l \in\left[x^{-1}(A \cap Y)\right] l \subseteq A^{\prime}$. Thus $A^{\prime}$ is left closed.

We define a map $\varphi: E_{X} \rightarrow E_{X}$ by $A \varphi=A^{\prime}, A \in E_{X}$, and we show that $\varphi$ is a non-injective surjective homomorphism.

(i) $\varphi$ is not injective.

It follows from the definition that $\left\{1, x, x^{2}\right\}, \varphi=\{1, x\}=\{1, x\} \varphi$ hence $\varphi$ is not injective.

(ii) $\varphi$ is surjective.

Let $A \in E_{X}$. Suppose that $A \cap Y=\varnothing$. Then it is immediate that $A \varphi=A$.

Now suppose that $A \cap Y \neq \varnothing$. Then $x, x^{2} \in A$. Let $B=(A \backslash Y) \cup\left\{x^{2}\right\} \cup[x(A \cap Y)]$. Obviously, $B$ is finite and nonempty. We show that $B$ is left closed. Let $w \in B$ and let $w^{\prime} \in R_{X}$ be such that $w^{\prime}<{ }_{1} w$. We have seen before that $A \backslash Y$ is left closed, so we can assume that $w \notin A \backslash Y$. Suppose that $w=x^{2}$. Since $A \cap Y \neq \varnothing$ and $A$ is left closed, we have $x^{2} \in A$ and so $w^{\prime} \in A \backslash Y \subseteq B$. Now suppose that $w=x^{3} u$ for some $u \in R_{X}$ such that $x^{2} u \in A$. We can assume that $w^{\prime}=x^{3} u^{\prime}$ and $u^{\prime}<_{l} u$ for some $u^{\prime} \in R_{X}$. Since $x^{2} u^{\prime}<_{l} x^{2} u$ and $A$ is left closed, we have $x^{2} u^{\prime} \in A$ and so $w^{\prime}=x^{3} u^{\prime} \in[x(A \cap Y)] \subseteq B$. Thus $B$ is left closed and so $B \in E_{X}$. It is immediate that $B \varphi=A$ and so $\varphi$ is surjective.

(iii) $\varphi$ is a homomorphism.

Let $A, B \in E_{X}$. Then $(A B) \varphi=[(A \cup B) \backslash Y] \cup\left(x^{-1}[(A \cup B) \cap Y]\right) l=(A \backslash Y) \cup(B \backslash Y) \cup$ $\left[x^{-1}(A \cap Y)\right] l \cup\left[x^{-1}(B \cap Y)\right]_{l}=(A \varphi)(B \varphi)$. Thus $\varphi$ is a homomorphism and the theorem is proved. 
Acknowledgements. This work was carried out while I held a research grant from the Calouste Gulbenkian Foundation, which I thank. I am also grateful to Professor W. D. Munn for all help and advice provided.

\section{REFERENCES}

1. M. Auslander and D. Buchsbaum, Groups, rings, modules (Harper \& Row 1974).

2. A. H. Clifford and G. B. Preston, The algebraic theory of semigroups (Math. Surveys 7 , Providence, R.I., Amer. Math. Soc., 1961 (vol. l) and 1967 (vol. II)).

3. J. M. Howle, Introduction to semigroup theory (Academic Press 1976).

4. W. D. MunN, Fundamental inverse semigroups, Quart. J. Math. Oxford (2)21 (1970), $157-170$.

5. W. D. MunN, Free inverse semigroups, Proc. London Math. Soc. (3)29 (1974), 385-404.

6. M. Petrich, Inverse semigroups (Wiley 1984).

7. N. R. ReIlly, Free generators in free inverse semigroups, Bull. Austral. Math. Soc. 7 (1972), 407-424; correction: ibid. 9 (1973), 479.

8. H. E. Scheiblich, Free inverse semigroups, Proc. Amer. Math. Soc. 38 (1973), 1-7.

Deparamento de Matemática

Universidade do Porto

4000 PORTO

Portugal
Department of Mathematics UNIVERSITY OF GLASGOW GLasGow G12 8QW 